\title{
EUVE Observations of BY Dra Systems
}

\author{
R. A. STERN ${ }^{1,3}$ AND J. J. DRAKE ${ }^{2}$ \\ ${ }^{1}$ Solar and Astrophysics Laboratory, Lockheed Martin Palo Alto Research Lab, \\ O/91-30 Bldg 252, 3251 Hanover Street, Palo Alto, CA, 94304, USA \\ ${ }^{2}$ Center for EUV Astrophysics, 2150 Kittredge Street, \\ University of California, Berkeley, CA 94720-5030, USA \\ ${ }^{3}$ Visiting Investigator, Center for EUV Astrophysics
}

We have observed 3 nearby BY Dra systems, FK Aqr, BF Lyn, and DH Leo, with the EUVE spectrometers. All 3 show evidence of high-temperature $\left(\sim 10^{7} \mathrm{~K}\right)$ plasma; FK Aqr and DH Leo show significant variability in their Deep Survey lightcurves. In FK Aqr, spectral differences between its "quiescent" and "active" states suggest possible differences in the plasma density. In $\mathrm{DH}$ Leo, the Deep Survey lightcurve, taken over nearly 8 days, shows a distinct period of $\sim 1.05$ days, consistent with the photometric period. The emission measure distributions of all three systems are rather similar in shape, and can be well-represented by a power law with slope $\sim 1.5$ from $6.2-7.0$ in $\log T$.

\section{Introduction}

The BY Draconis stars are a group of red ( $\mathrm{dKe}-\mathrm{dMe}$ ) low-amplitude variables with photometric periods of typically a few days, Ca II and often $\mathrm{H} \alpha$ emission, and unusually bright X-ray emission for their spectral type, with a typical $L_{x} \approx 5 \times 10^{29} \mathrm{erg} \mathrm{s}^{-1}$ (Bopp \& Fekel 1977; Caillault 1982). Most, but not all, are binaries in short period (few days) orbits, with rapid rotation the crucial factor in their high level of activity. A few are dMe flare stars (Bopp \& Fekel 1977; Strassmeier et al. 1988). From the results of EUV sky surveys (Pounds et al. 1993; Bowyer et al. 1994), it now appears that many of the BY Dra systems are strong EUV emitters as well: these include KZ And, FK Aqr, V1396 Cyg, V775 Her, DH Leo, BF Lyn, YY Gem, V833 Tau, CC Eri, and BY Dra, the prototypical system. All of these systems are within $\approx 30 \mathrm{pc}$ of the Sun. Because they are relatively young main sequence stars, with X-ray activity levels typically $10^{2}$ or more times solar, they represent some of our most accessible main sequence stellar laboratories in the EUV with which to examine the effect of strong magnetic heating on coronal temperature. We have performed a detailed study of 3 representative, nearby, EUV-bright BY Dra systems: FK Aqr, DH Leo, and BF Lyn. The characteristics of these systems are listed in Table 1 . The column densities $\left(N_{\mathrm{H}}\right)$ to these systems are uncertain, but are probably $\lesssim 1-2 \times 10^{18} \mathrm{~cm}^{-2}$.

\section{The FK Aqr Lightcurves}

The Deep Survey (DS) telescope feeds both an imaging detector and the 3 EUVE spectrometers (Bowyer \& Malina 1991), thus providing simultaneous photometry and spectrophotometry. Sources near telescope boresight are imaged in the DS/Lexan band (70-180 ). In Figure 1 we show the lightcurves obtained, in DS/Lexan and by summing the 80-140 Åregion in the SW spectrometer. The data are binned over $\approx 1$ satellite orbit, with $\sim 2 \mathrm{ks}$ effective exposure time in each bin. The light curves are quite similar (the DS/Lexan has smaller errors due to the higher count rate), even to the extent of recording a brief flare at $\sim 135 \mathrm{ks}$ into the observation. The most striking aspect of the lightcurves is the dramatic rise in EUV flux at $\sim 220 \mathrm{ks}$ into the observation. Since FK Aqr is not an eclipsing system, we may attribute this rise either to rapid growth of a 
TABLE 1. BY Dra Systems Observed (Lex/B and Al/C are $E U V E$ scanner count rates ks ${ }^{-1}$ )

$\begin{array}{rlrrrr}\text { Star } & \text { Sp. Type } & \text { Porb }_{\text {or }} & d(\mathrm{pc}) & \text { Lex/B } & \text { Al/C } \\ \text { FK Aqr } & \text { dM2e/dM2e } & 4.08 & 7.7 & 150 & 50 \\ \text { BF Lyn } & \text { K2 V/dK } & 3.8 & 29 & 90 & 50 \\ \text { DH Leo } & \text { K0/K7/K5 } & 1.07 & 33 & 50 & 40\end{array}$

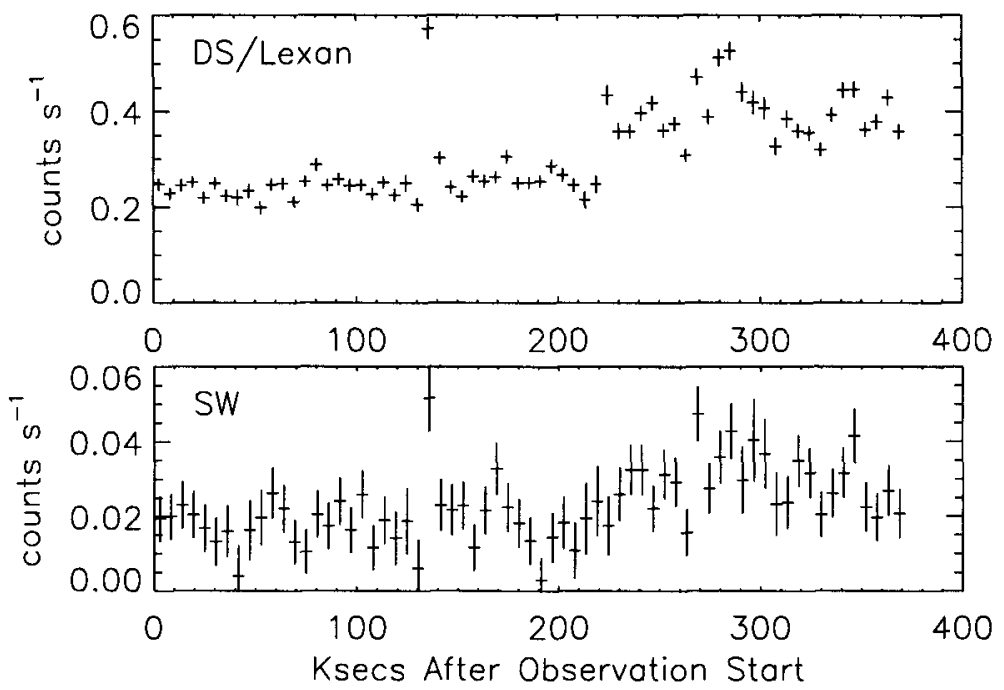

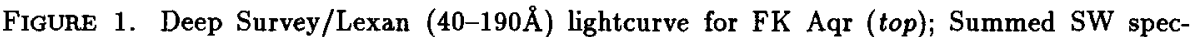
trometer (80-140 $\AA$ ) lightcurve (bottom). Each data point covers about 1 spacecraft orbit (96 mins.)

large active region or complex of active regions, or perhaps to the rotation of an already existing active region complex onto the visible disk of one of the stars in the system. Since the length of the observation $(\approx 4.28 \mathrm{~d})$ is slightly longer than the orbital period $(4.08 \mathrm{~d})$, but slightly shorter than the photometric period $(4.39 \mathrm{~d})$, we cannot rule out either explanation or a combination of both.

\section{The FK Aqr Spectra}

Given the appearance of "quiescent" and "active" states in the FK Aqr lightcurve, we have extracted EUVE short wavelength and medium wavelength spectra in two parts: one including the period before the lightcurve "transition" (but excluding the flare at 135 $\mathrm{ks}$ ), and a second part including both the flare and the time period after the transition. These are shown in Figure 2. The most noticeable differences between the spectra are the relative strengths of the Fe XXI 128.7 $\AA$ line compared to the other Fe XXI line and lines from nearby ionization stages. This could be an indicator of higher $\left(\sim 10^{13} \mathrm{~cm}^{-3}\right)$ densities; however, an independent confirmation through the Fe XXII density-sensitive $117 / 114$ line ratio is problematic because of the lower statistical significance of these line fluxes. 

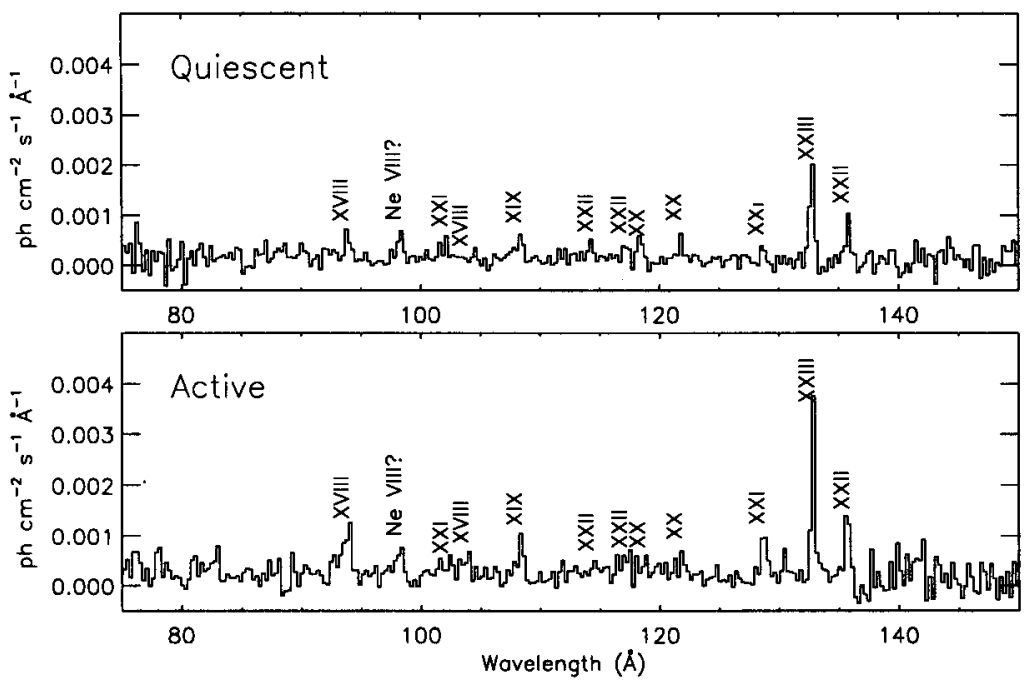

FIGURE 2. Quiescent (top) and active (bottom) SW spectra for FK Aqr. The data have been rebinned by a factor of 4 to about $0.26 \mathrm{~A}$. The effective exposure times are 74 and $54 \mathrm{ks}$, respectively. In each plot, the ionization stage of $\mathrm{Fe}$ for each line is indicated.

\section{DH Leo}

\subsection{The Lightcurve: Rotational Modulation?}

Our observation of DH Leo covered a time span of $\approx 670 \mathrm{ks}$, or over 7 binary or photometric periods. The DS/Lexan lightcurve for this observation is shown in Figure 3, along with a periodogram analysis (using the "fast" algorithm of Press \& Rybicki 1989). The most significant peak occurs at a period of $\approx 1.05$ days. Because this period is close to $\sim 1$ day as well as the photometric period of 1.067 days (Barden et al. 1986), we also performed a periodogram analysis of the detector background, but failed to find a significant peak at either of these values. Thus, for the moment we conclude that the periodicity is real, and is close enough (within an estimated error of $\pm 0.05 \mathrm{~d}$ ) that we may associate it with the photometric period of DH Leo. A phased lightcurve at this period is shown in Figure 4, indicating a definite "ripple" in the EUV flux. No other obvious periodicity in the $E U V E$ satellite at $\sim 1$ day is known to produce such a variation in the source count rate, thus we tentatively conclude that our lightcurve is an indication of rotational modulation in the DH Leo system.

\subsection{Spectrum}

Dividing up the DH Leo spectrum by phase into "low level" and "high level" count rate did not produce significant spectral differences, unlike in the case of FK Aqr. The relative strengths of the Fe XXII 117/114 line ratio are consistent with the low-density limit (i.e. $\left.\lesssim 10^{12} \mathrm{~cm}^{-3}\right)$. 

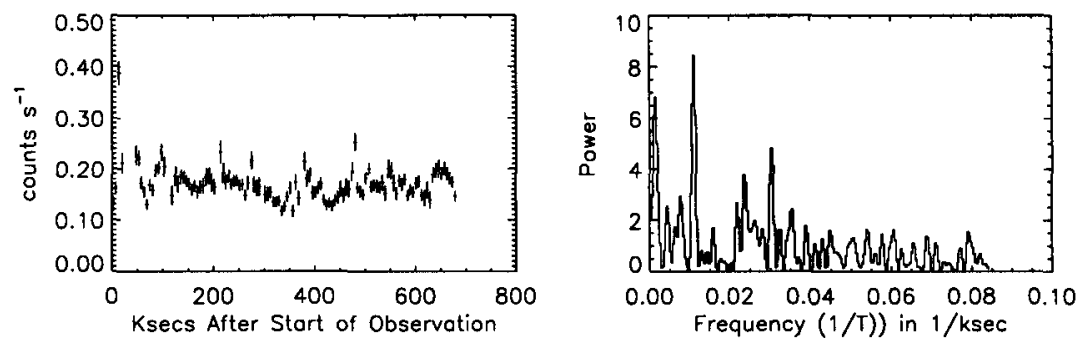

Figure 3. DS/Lexan lightcurve for DH Leo (left) and periodogram analysis (right)

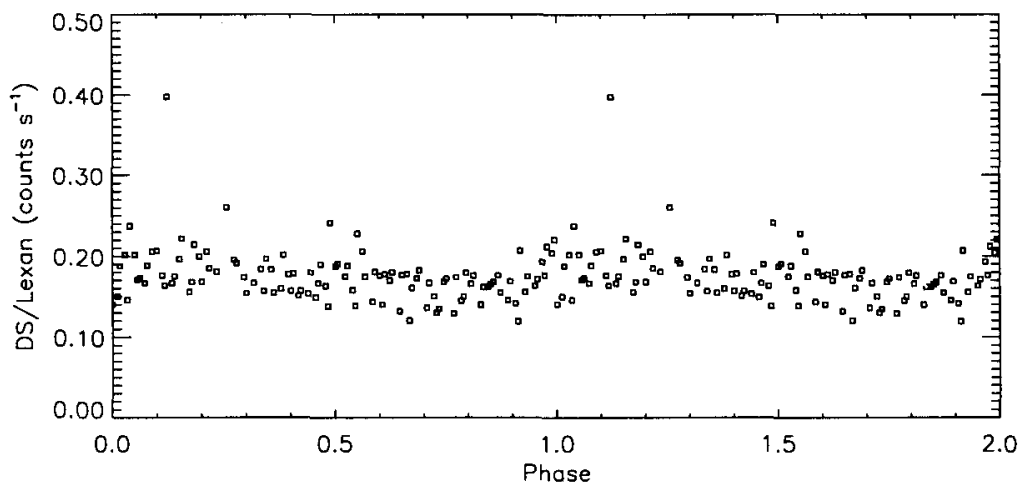

Figure 4. Phased lightcurve for $\mathrm{DH}$ Leo (phase $=0$. has been arbitrarily chosen at the start of the observation)

\section{The BF Lyn Lightcurve and Spectrum}

The BF Lyn DS/Lexan lightcurve exhibited variability of up to $50 \%$ during the course of the observation $(\approx 2.5 \mathrm{~d})$. Since the total observation coverage extends for less than a single orbital or photometric period of the system ( 3.8 days), the remaining variability is probably due to a combination of flares, rotational modulation, or growth and decay of active regions. BF Lyn is a relatively weak EUV source, and the $70 \mathrm{ks}$ effective exposure time reveals fewer lines (many at low significance) than the other 2 BY Dra systems. However, the Fe XXIII/XX feature is prominent, and other ionization stages from XV-XXII are also present, thus indicating a relatively hot corona.

\section{Emission Measure Distributions}

In Figure 5 we show preliminary emission measure distributions for the BY Dra systems. These have been computed using the Fe emissivities of Brickhouse et al. (1995), and the "Pottasch" method of using the peak temperature of the emissivity function. As such they represent a quick estimate of the form of the EM distribution; further analysis using spectral synthesis methods may yield a better estimate of the EM. Note that overall shapes of the EM distributions are quite similar for all the systems, and in the range $\log T=6.2-7.0$, can be well approximated by a power law of slope $\sim 1.5$. DH Leo, the shortest period system, also appears to have the greatest quantity of the highest 


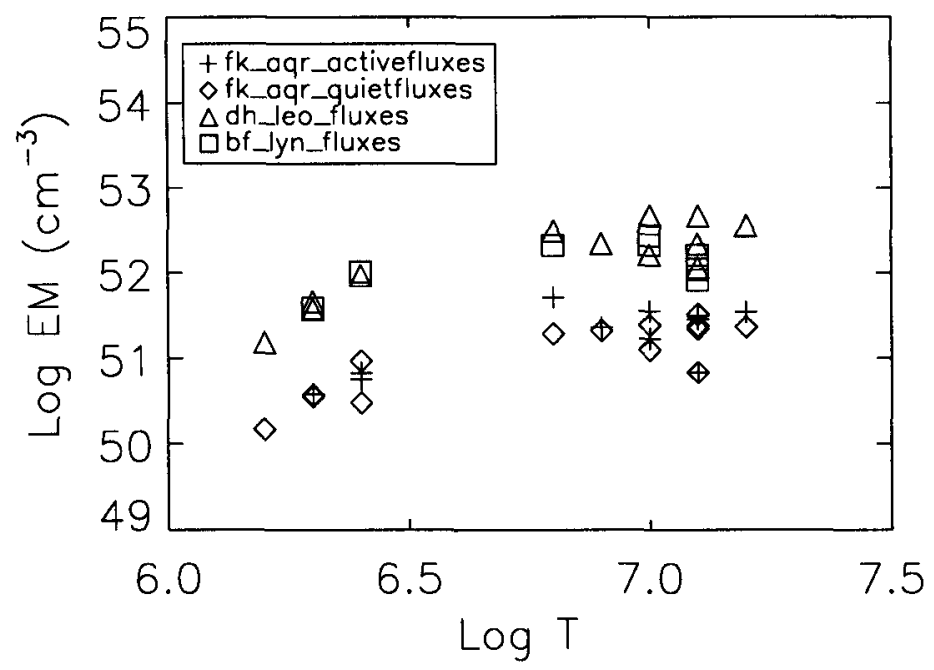

Frgure 5. Emission measure distributions for the BY Dra systems (see text)

temperature material. Even allowing for the scatter in the plot due to a combination of statistical uncertainties (up to a factor of 2 for the weaker lines), and atomic physics uncertainties, the enhancement in the EM at $\log \mathrm{T} \sim 6.8$ as seen in the Fe XVIII 93.9 Aline for the "active" FK Aqr spectrum is still quite significant, and somewhat reminiscent of the "bump" seen in the Capella EM distribution in Dupree et al. (1993) and Brickhouse et al. (1995). We note that deriving the EM for the quiescent spectrum assuming $n_{e}=$ $10^{13} \mathrm{~cm}^{-3}$ made only a slight difference in the EM distribution.

\section{Summary}

$E U V E$ observations provide one of the best ways to study both the variability and the temperature structure of the coronae of BY Dra systems. Both periodic modulations and stochastic variability are amply evident in the short wavelength high temperature lines seen by EUVE: the DH Leo study for over 7 binary orbits is probably the best example to date of rotational modulation of stellar coronal emission. In addition, there is a hint of a high density structure in the quiescent corona of FK Aqr as compared to the "active" or flaring corona.

R.A.S. wishes to thank the scientists and staff of the Center for EUV Astrophysics for making his stay there enjoyable and productive. R.A.S. was supported in part by NASA contract NAS5-32640 and by the Lockheed Independent Research Program. J.J.D. was supported by NASA Grant AST91-15090. 


\section{REFERENCES}

Barden, S. C., Ramsey, L. W., Fried, R. E, Guinan, E. F., \& Wacker, S. W. 1986, in Cool Stars, Stellar Systems, and The Sun, ed. M. Zeilik \& D.M. Gibson, Berlin: Springer, 291

BopP, B. W. \& FeKel, F. C., JR. 1977, AJ, 82, 490

Bowyer, S., Lieu, R., Lampton, M, Lewis, J., Wu, X., Drake, J. J., \& Malina, R. F. 1994, ApJS, 93, 569

Bowyer, S. \& Malina, R. F. 1991, in Extreme Ultraviolet Astronomy, ed. R.F. Malina \& S. Bowyer, New York: Pergamon Press, 397

Brickhouse, N. S., RaYmond, J. C., \& SMith, B. W. 1995, ApJS, in press

Canlault, J. P. 1982, AJ, 87, 558

Dupree, A. K., Brickhouse, N. S., Doschek, G. A., Green, J. C., \& Raymond, J. C. 1993, ApJ, 418, L41

Pounds, K. A., ET AL. 1993, MNRAS, 260, 77

Press, W. H. \& Rybicki, G.B. 1989, ApJ, 338, 277

STrASSMEIER, K. G., ET AL. 1988, A\&AS, 72, 291 